\title{
Patient selection for LIVE therapy - From clinical indications to multimodality imaging individual case planning
}

\author{
Paulo Neves ${ }^{1}$, Thasee Pillay ${ }^{2}$, Lon Annest ${ }^{2}$, Kevin Van Bladel ${ }^{2}$, Erhard Kaiser ${ }^{3}$, Fabian \\ Stahl $^{4}$, Thorsten Hanke ${ }^{5}$, Martin Swaans ${ }^{6}$, Patrick Klein ${ }^{6}$, Tobias Ruf ${ }^{7}$, and Ralph Stephan \\ von Bardeleben ${ }^{7}$ \\ ${ }^{1}$ Centro Hospitalar de Vila Nova de Gaia Espinho EPE \\ ${ }^{2}$ BioVentrix Inc \\ ${ }^{3}$ Private Practice for Internal Medicine and Cardiology \\ ${ }^{4}$ Kardiologie am Tibarg \\ ${ }^{5}$ Asklepios Hospital Harburg \\ ${ }^{6}$ St.Antonius Hospital \\ ${ }^{7}$ Universitätsmedizin Mainz
}

February 13, 2021

\begin{abstract}
Background LIVE (Less Invasive Ventricular Enhancement) with Revivent TC is an innovative therapy for symptomatic ischemic Heart Failure (HF). It is designed to reconstruct a negatively remodeled left ventricle (LV) after an anterior myocardial infarction (MI) by plication of the scar tissue. Its indications are specific and, as with any other structural heart intervention, the success of the procedure starts with appropriate patient selection. We aim to present the indications of the technique, crucial aspects in patient selection and individual case planning approach. Methods and Results After clinical evaluation, transthoracic echocardiography is the first imaging modality to be performed in a potential candidate for the therapy. However, definitive indication and detailed case planning rely on late gadolinium-enhanced cardiac magnetic resonance imaging or multiphasic contrast-enhanced cardiac computed tomography. These imaging modalities also assist with relative or absolute contra-indications for the procedure. Individual assessment is done to tailor the procedure to the specifics of the LV anatomy and location of the myocardial scar. Conclusion LIVE procedure is a unique intervention to treat symptomatic heart failure and ischemic cardiomyopathy after anterior MI. It is a highly customizable intervention that allows a patient-tailored approach, based on multimodality imaging assessment and planification.
\end{abstract}

\section{REVIEW ARTICLE}

\section{PATIENT SELECTION FOR LIVE THERAPY}

\section{From clinical indications to multimodality imaging individual case planning}

Paulo Neves ${ }^{1}$, Thasee Pillay ${ }^{2}$, Lon Annest ${ }^{2}$, Kevin van Bladel ${ }^{2}$, Erhard Kaiser ${ }^{3}$, Fabian Stahl ${ }^{4}$, Thorsten Hanke $^{5}$, Martin Swaans ${ }^{6}$, Patrick Klein ${ }^{7}$, Tobias Ruf ${ }^{8}$, Ralph Stephan von Bardeleben ${ }^{8}$

${ }^{1}$ Cardiothoracic Surgery Department, Centro Hospitalar de Vila Nova de Gaia/Espinho, Vila Nova de Gaia, Portugal

2 BioVentrix Inc., 12647 Alcosta Boulevard, Suite 400 San Ramon, CA 94583, USA

${ }^{3}$ Private Practice for Internal Medicine and Cardiology, Frankfurt am Main, Germany 
${ }^{4}$ Kardiologie am Tibarg, Hamburg, Germany

${ }^{5}$ Department of Cardiac Surgery, Asklepios Hospital Harburg, Hamburg, Germany

${ }^{6}$ Department of Cardiology, St. Antonius Hospital, Nieuwegein, Netherlands

${ }^{7}$ Department of Cardiothoracic Surgery, St. Antonius Ziekenhuis, Nieuwegein, Netherlands

${ }^{8}$ Heart Valve Center Mainz, Center of Cardiology, Cardiology I, University Medical Center Mainz, Mainz, Germany

Contact:

Paulo Neves

Serviço de Cirurgia Cardiotorácica do Centro Hospitalar de Vila Nova de Gaia/Espinho

Rua Conceição Fernandes, s/n

4434-502 VN Gaia

Tel. +351227865100

Email: pauloacn@gmail.com

Keywords: LIVE, Revivent TC, ventricular restoration, heart failure

Word count (abstract): 197

Word count: 2076

\section{Abstract \\ Background}

LIVE (Less Invasive Ventricular Enhancement) with Revivent TC is an innovative therapy for symptomatic ischemic Heart Failure (HF). It is designed to reconstruct a negatively remodeled left ventricle (LV) after an anterior myocardial infarction (MI) by plication of the scar tissue. Its indications are specific and, as with any other structural heart intervention, the success of the procedure starts with appropriate patient selection. We aim to present the indications of the technique, crucial aspects in patient selection and individual case planning approach.

\section{Methods and Results}

After clinical evaluation, transthoracic echocardiography is the first imaging modality to be performed in a potential candidate for the therapy. However, definitive indication and detailed case planning rely on late gadolinium-enhanced cardiac magnetic resonance imaging or multiphasic contrast-enhanced cardiac computed tomography. These imaging modalities also assist with relative or absolute contra-indications for the procedure. Individual assessment is done to tailor the procedure to the specifics of the LV anatomy and location of the myocardial scar.

\section{Conclusion}

LIVE procedure is a unique intervention to treat symptomatic heart failure and ischemic cardiomyopathy after anterior MI. It is a highly customizable intervention that allows a patient-tailored approach, based on multimodality imaging assessment and planification.

\section{Background}

Heart failure (HF) is an increasingly important source of morbidity and mortality in developed nations, and ischemic cardiomyopathy (ICM) is the most common cause. ${ }^{[1]}$ Despite adequate treatment, with prompt percutaneous coronary intervention (PCI), up to $50 \%$ of patients lose $18 \%$ or more of left ventricular (LV) mass due to an acute myocardial infarction (MI). ${ }^{[2]}$ Following primary PCI, guideline-directed medical therapy 
(GDMT) decreases the degree of LV remodelling, consequently reducing the development of ICM. Notwithstanding optimal GDMT after early reperfusion therapy, negative LV remodelling (defined as an increase of LV end-diastolic volume $>20 \%$ from baseline) has been observed in approximately one third of acute MI patients. ${ }^{[3]}$ This increased LV volume is associated with major cardiac events, such as congestive HF, functional mitral regurgitation (FMR), apical aneurysm and risk of ventricular arrhythmia and sudden cardiac death. It is also related with decreased survival. ${ }^{[4]}$

In patients with a negatively remodeled LV, in whom the LV volume is increased, LV ejection fraction (EF) is depressed because of increased wall tension and less efficient myocardial fiber orientation and anterior myocardial scar tissue of at least $50 \%$ transmurality, surgical ventricular reconstruction (SVR) is an established therapy that can be considered. Its drawbacks are that this is a highly invasive open-heart surgical procedure performed with the use of extracorporeal circulation (ECC) and cardioplegic myocardial arrest. ${ }^{[5]}$ Less Invasive Ventricular Enhancement (LIVE) technique with Revivent TC system (BioVentrix Inc., San Ramon, CA, USA) has been developed as an equally effective therapy to reconstruct the LV to decrease LV volume, reconstruct its physiologic shape and reduce LV wall tension. All this is aimed to improve LV performance leading to reduction of HF symptoms, increase quality of life and improve survival. ${ }^{[6]}$

\section{LIVE therapy overview}

LIVE therapy is a hybrid procedure, based on teamwork between a cardiac surgeon, an interventional cardiologist and an imaging cardiologist/radiologist. It is performed in a hybrid operative room (OR) under general anesthesia. It requires a small left thoracotomy and, depending on the strategy, a right internal jugular vein access (RIJV). The procedure is guided by multimodality imaging, with constant fluoroscopic and transesophageal echocardiography (TEE) monitoring.

LV shape and size are restored on a beating heart, without ECC by plication and exclusion of the scarred myocardium. This is achieved by implantation of a series of titanium microanchors $(5 \mathrm{~mm} \times 25 \mathrm{~mm})$ brought together over a poly-ether-ether-ketone (PEEK) tether $(1.7 \mathrm{~mm} \times 1.0 \mathrm{~mm})$ to exclude the scarred myocardium. This is either achieved as a LV-LV approximation, or as a RV-LV approximation, depending on the scar distribution. In the RV-LV approach, internal anchors are deployed over-the-wire, through the RIJV, on the right side of the ventricular septum (Figure 1 ).

The LIVE procedure most recent results were presented at the European Society of Cardiology 2020 Congress. ${ }^{[7]}$ No early or intra-hospital mortality was reported. A mean of 2.3 anchor pairs (median 2) were used to reshape the LV. Echocardiographic data showed an increase in LV ejection fraction (EF) from $31.4+-9.2 \%$ to $40.0+-12.4 \%$ (change $+29.8 \%, \mathrm{p}<0.001$ ) and LV end-systolic volume index (LVESVI) reduction from $66.6+-29.3 \mathrm{ml} / \mathrm{m} 2$ to $40.7+-21.5 \mathrm{ml} / \mathrm{m} 2$ (change $-38 \%, \mathrm{p}<0.001$ ) and $\mathrm{LV}$ end-diastolic volume index (LVEDVI) reduction from $92.8+-39.2 \mathrm{ml} / \mathrm{m} 2$ to $60.6+-25.9 \mathrm{ml} / \mathrm{m} 2$ (change $-33.2 \%, \mathrm{p}=0.001$ ) after the procedure. In the mean follow-up period of 9.8 months, NYHA class improved a median of 1 grade and there was no late mortality.

Recently, to robustly demonstrate that the LIVE procedure with the Revivent TC system is more effective than GDMT alone for the treatment of ischemic HF, a randomized controlled trial - Revivent TC versus Guideline Determined Medical Therapy (REVIVE-HF, NCT03845127) - has been developed and started patient enrolment in 2019. Results are expected in 2022.

\section{Patient screening}

Potential candidates for LIVE therapy present with symptomatic HF (NYHA class $\geq$ II) due to cardiac dysfunction caused by a previous anterior MI, resulting in increased LV systolic volume and in a discrete, contiguous, acontractile, (akinetic and/or dyskinetic) scar located in the antero-septal, apical region of the left ventricle. The myocardial scar needs to be at least $50 \%$ transmural to provide sufficient support and resistance to the plicating anchors. Alternative myocardial scar locations can also be considered for treatment, as lateral wall scar. Furthermore, patients with purely dyskinetic LV anterior or antero-apical walls, that could be described as classic or "true" LV aneurysms are good candidates for LIVE therapy. 
There should be sufficient scar maturation or fibrotic tensile strength developed to provide adequate support (and prevent "pull through" and wall lesion) for the anchors. As such, the initial ischemic myocardial event should have taken place at least 90 days before the intervention. After appropriate clinical assessment, imaging is essential to assess indication, eligibility and potential contra-indications for the therapy (Table 1 - Multi-modality imaging overview; Table 2- Exclusion criteria). This stepwise approach is described below.

\section{Transthoracic echocardiography}

Initial assessment includes transthoracic echocardiography (TTE), where the focus is to look for akinetic and/or dyskinetic areas within the left anterior descending (LAD) artery myocardial territory - LV anterior wall, anterior septum and apex. For that purpose, the apical 2-, 3- and 4 -chamber-views, respectively, are most commonly used. Standard echocardiographic equipment is used, including harmonic imaging with a $3.5 \mathrm{MHz}$ broadband transducer, with capture of at least three beats per view ( 5 beats/view in atrial fibrillation at a normal heart rate). Additionally, contractility of the basal segments is assessed, in order to estimate both the baseline residual function after the reconstruction and - more importantly - the potential for improved myocardial performance. Valvular heart disease and right ventricular (RV) size and function are also evaluated. A contrast-enhanced examination is not mandatory, although it further increases diagnostic accuracy of intracardiac thrombus, which is a relative contra-indication for the procedure. Figure 2 summarizes key aspects in TTE screening for the LIVE procedure.

\section{Cardiac magnetic resonance imaging}

If the patient is considered suitable for the technique by clinical and initial TTE evaluation, a late gadolinium contrast enhanced (LGE) cardiac magnetic resonance (CMR) is requested in order to precisely define the location and transmurality of the myocardium scar. Furthermore, LGE CMR gives important information about LV geometry, volume and function and it accurately rules out the presence of intracardiac thrombus. If a thrombus is detected, the recommendation is to anticoagulate patient with vitamin-K antagonizing agents, e.g. warfarin, for three months and repeat the LGE CMR. Figure 3summarizes key aspects in LGE CMR screening for the LIVE procedure.

\section{Cardiac computed tomography}

Alternatively to LGE CMR, in patients with intracardiac devices not compatible with it, LV wall motion and scar assessment is done using contrast enhanced multiphasic 4D computed tomography (CT). Even in patients with MRI conditional devices, 4D CT may be better for regional motion assessment of the interventricular septum, mainly in patients with pacemaker / implantable cardioverter defibrillator (ICD) / cardiac resynchronization therapy (CRT) leads placed in the right side of it. Despite not having direct scar determination capabilities, 4D multiphasic CT scan is a powerful tool in regional wall thickness and motion assessment as well as LV volume assessment. ${ }^{[8]}$ Thinned, akinetic or dyskinetic regions are considered non-viable and, as such, suitable for exclusion with the LIVE technique. Although sensitivity and specificity are lower than LGE CMR, existence of an apical thrombus can also be assessed by CT. ${ }^{[9]}$ Extensive LV wall calcification is an absolute contraindication for the procedure and can be easily depicted from CT, and not from CMR. Additionally, 3D multiphasic volume rendering is an important tool in determining scar location, assessing basal wall contractility and precise planning of anchor placement. Finally, 4D CT is useful in assessing graft positioning in post-coronary artery bypass graft (CABG) patients. Figure 4 summarizes key aspects in $4 \mathrm{D}$ multiphasic CT screening for the LIVE procedure.

\section{Case planning}

LIVE therapy with Revivent TC encompasses 2 main techniques: RV-LV and LV-LV approaches. The first one, RV-LV, includes an internal anchor deployment, through the RIJV, bringing the interventricular septum together with the antero-lateral LV wall (Figure 1). The more distal apex is also excluded with external, surgically applied anchor pairs. ${ }^{[10]}$ When the septal component is not significant or transmural enough, antero-lateral and apical scar is excluded with external, surgically applied, LV-LV anchor pairs. 
RV-LV approach is used when there is a significant septal distribution of the myocardial scar. To evaluate the scar location and to plan the procedure, short axis stacks of LGE CMR or 4D multiphasic CT scan are used. Both location and extension of the scar are assessed in order to plan anchor placement and the number of needed devices. The internal anchor and the corresponding external anchor pair are the first ones to be implanted. Then, if more scar is identified basally to this pair, a second external anchor pair is applied in this location. This anchor pair can exclude a portion of septal scar as well, sacrificing a small portion of the right ventricle - this is nicknamed the "Antonius stitch", after the development of this modification at the St. Antonius Hospital, Nieuwegein, The Netherlands (a hospital with extensive experience with the LIVE procedure) (Figure 5 ). Finally, the apical component is treated with LV-LV anchor pairs. If the scar doesn't affect the entire apical circumference, anchor placement can be tailored to exclude only the non-viable segments.

\section{$L V-L V$}

LV-LV scar exclusion can be applied when there is LV scar limited to the antero-lateral and apical walls, without septal involvement. Consequently, the reconstruction doesn't include the septum in the exclusion. As such, the procedure is carried out in a purely surgical fashion.

Case planning follows the same principles of multimodality imaging. Anchor deployment and orientation can be tailored to the scar location and full scar exclusion should be feasible in most patients (Figure 6B ). The patients treated with this therapy have suffered from a LAD MI and the consequent scar tissue is located in anterior, antero-septal, antero-lateral and apical segments. However, different scar locations can be addressed with this technique as it is essentially based on plicating scarred segments, using the same surgical approach. In fact, manipulation of the heart from an apical skin incision by the liberal use of "myocardial leashes" - temporary "U" stitches in the scarred myocardium, snugged and fixed with a hemostatic clamp, enabling direct manipulation of the heart without hemodynamic disturbance - allows the surgeon to expose the lateral and inferior walls as well. Figure $\mathbf{6 C}$ shows an example of a post-operative left ventriculography of patient with an extensive lateral scar due to a previous left circumflex myocardial infarction.

\section{Conclusion}

LIVE procedure is a hybrid and minimally-invasive procedure to treat a negatively remodeled LV by plication of myocardial scar tissue in the context of ischemic cardiomyopathy after an anterior MI. Its indications are specific and patient selection is crucial to achieve an optimal result. After clinical assessment, multimodality imaging is essential to confirm indication, rule-out contraindications and to plan the procedure. Since the procedure is highly customizable by the combination of its different technical options, it allows for a true patient tailored approach to achieve the optimal results in improving ventricular performance.

\section{Figure legends}

Figure 1 - Internal and external anchor deployment.

Figure 2-A \& B - apical 2- \& 4-chamber views, respectively, showing LV dilatation and bulbous apex, with apical, distal anterior and septal wall thinning; C \& D-apical 4-chamber view showing apical thrombus without (C) and with contrast-enhancement (D)

Figure 3 - A \& B - 2 \& 4 chamber LGE views showing LV dilatation and transmural scar in distal antero-septal walls and apex; C \& D - Large LV apical thrombus

Figure 4 - Contrast-enhanced multiphasic CT scan - 2- (A) \& 4-chamber view (B), demonstrating LV dilatation, anterior and apical wall thinning (blue arrows) and LV apical thrombus $\left(^{*}\right)$; $\mathrm{C}-$ short axis view of the same patient, demonstrating antero-septal wall thinning (blue arrows); D - 3D volume rendering 
contrast-enhanced CT scan of a patient with extensive LV antero-lateral scar and wall calcification (white arrows). Pacemaker leads are illustrated in (B) and (D) ().

Figure 5 - CT-Scan - A - case planning according to scar location on 3D volume rendering; B - RV-LV basal "Antonius" stitch, addressing additional antero-lateral and septal components of the scar; C - expected result of pre-operative LGE CMR (red line). Anchors are represented by yellow rectangles.

Figure 6 - A - pre-operative 3D volume rendering CT of a patient with distal antero-lateral and apical scar; B - post-operative 3D volume rendering CT of the same patient with $3 \mathrm{LV}-\mathrm{LV}$ anchor pairs deployed, demonstrating full scar exclusion; C - post-operative LV ventriculography of patient with lateral LV scar, treated with 4 linear LV-LV anchor pairs

\section{Author contributions}

PN and TP did paper drafting. All other authors did critical revision of the article and approved the article.

\section{References}

1. Roth GA, Mensah GA, Johnson CO, et al; GBD-NHLBI-JACC Global Burden of Cardiovascular Diseases Writing Group. Global Burden of Cardiovascular Diseases and Risk Factors, 1990-2019: Update From the GBD 2019 Study. J Am Coll Cardiol. 2020 Dec 22;76(25):2982-3021.

2. Stone GW, Selker HP, Thiele H, Patel MR, Udelson JE, Ohman EM, Maehara A, Eitel I, Granger CB, Jenkins PL, Nichols M, Ben-Yehuda O. Relationship Between Infarct Size and Outcomes Following Primary PCI: Patient-Level Analysis From 10 Randomized Trials. J Am Coll Cardiol. 2016 Apr 12;67(14):1674-83. doi: 10.1016/j.jacc.2016.01.069. PMID: 27056772.

3. Savoye C, Equine O, Tricot O, et al. Left ventricular remodeling after anterior wall acute myocar- dial infarction in modern clinical practice (from the REmodelage VEntriculaire [REVE] study group). Am J Cardiol. 2006;98(9): 1144-9.

4. Bolognese L, Neskovic AN, Parodi G, et al. Left ventricular remodeling after primary coronary angioplasty: patterns of left ventricular dilation and long-term prognostic implications. Circulation. 2002;106(18):2351-7.

5. Ruzza A, Czer LSC, Arabia F, Vespignani R, Esmailian F, Cheng W, De Robertis MA, Trento A. Left Ventricular Reconstruction for Postinfarction Left Ventricular Aneurysm: Review of Surgical Techniques. Tex Heart Inst J. 2017 Oct 1;44(5):326-335.

6. Zivelonghi C, Klein P, Swaans MJ, Agostoni P. Hybrid transcatheter left ventricular reconstruction for the treatment of ischaemic cardiomyopathy. EuroIntervention. 2018 Mar 20;13(16):1899-1901.

7. P Neves, T Pillay, K Van Bladel, L Annest. LIVE procedure for ischemic heart failure: the evolution of an unique technique. European Heart Journal, Volume 41, Issue Supplement_2, November 2020, ehaa946.1132

8. Rizvi A, Deaño RC, Bachman DP, Xiong G, Min JK, Truong QA. Analysis of ventricular function by CT. J Cardiovasc Comput Tomogr. 2015 Jan-Feb;9(1):1-12.

9. McCarthy CP, Vaduganathan M, McCarthy KJ, Januzzi JL Jr, Bhatt DL, McEvoy JW. Left Ventricular Thrombus After Acute Myocardial Infarction: Screening, Prevention, and Treatment. JAMA Cardiol. 2018 Jul 1;3(7):642-649.

10. Pillay T, Neves P, Benetti F, Van Bladel K, Wechsler A, Annest L. Minimal access left ventricular reconstruction. J Card Surg. 2020 Nov 1. 

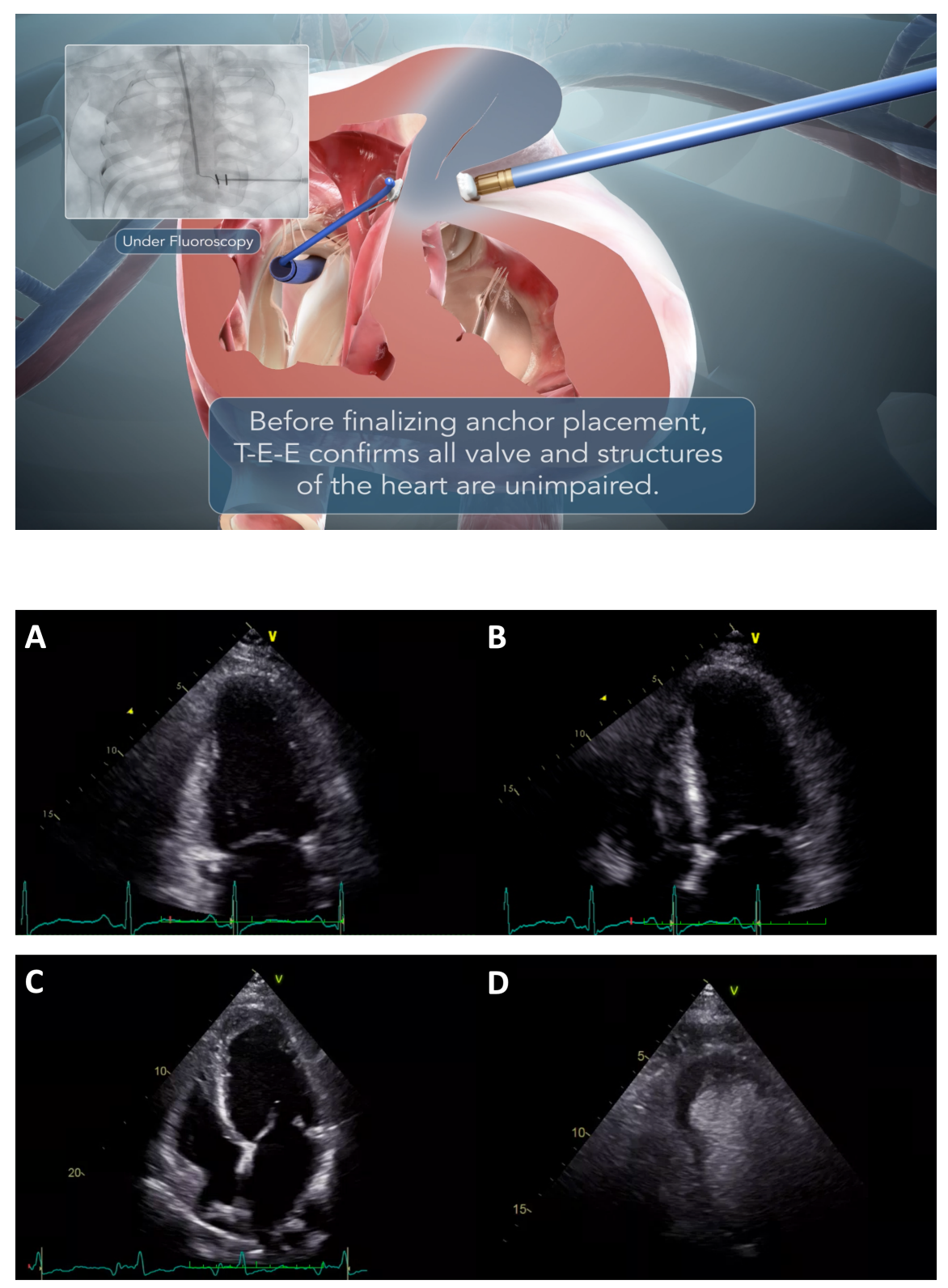

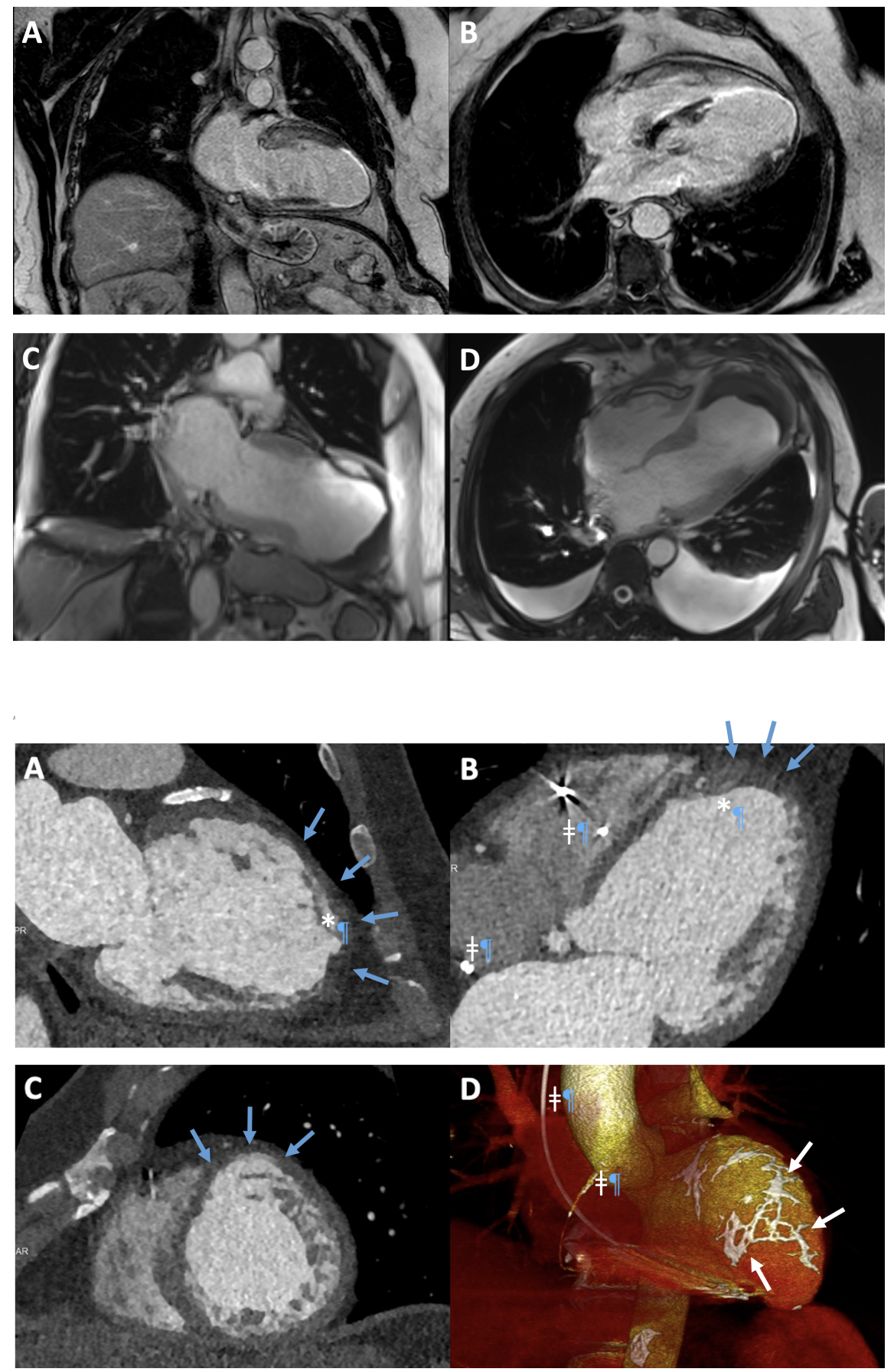

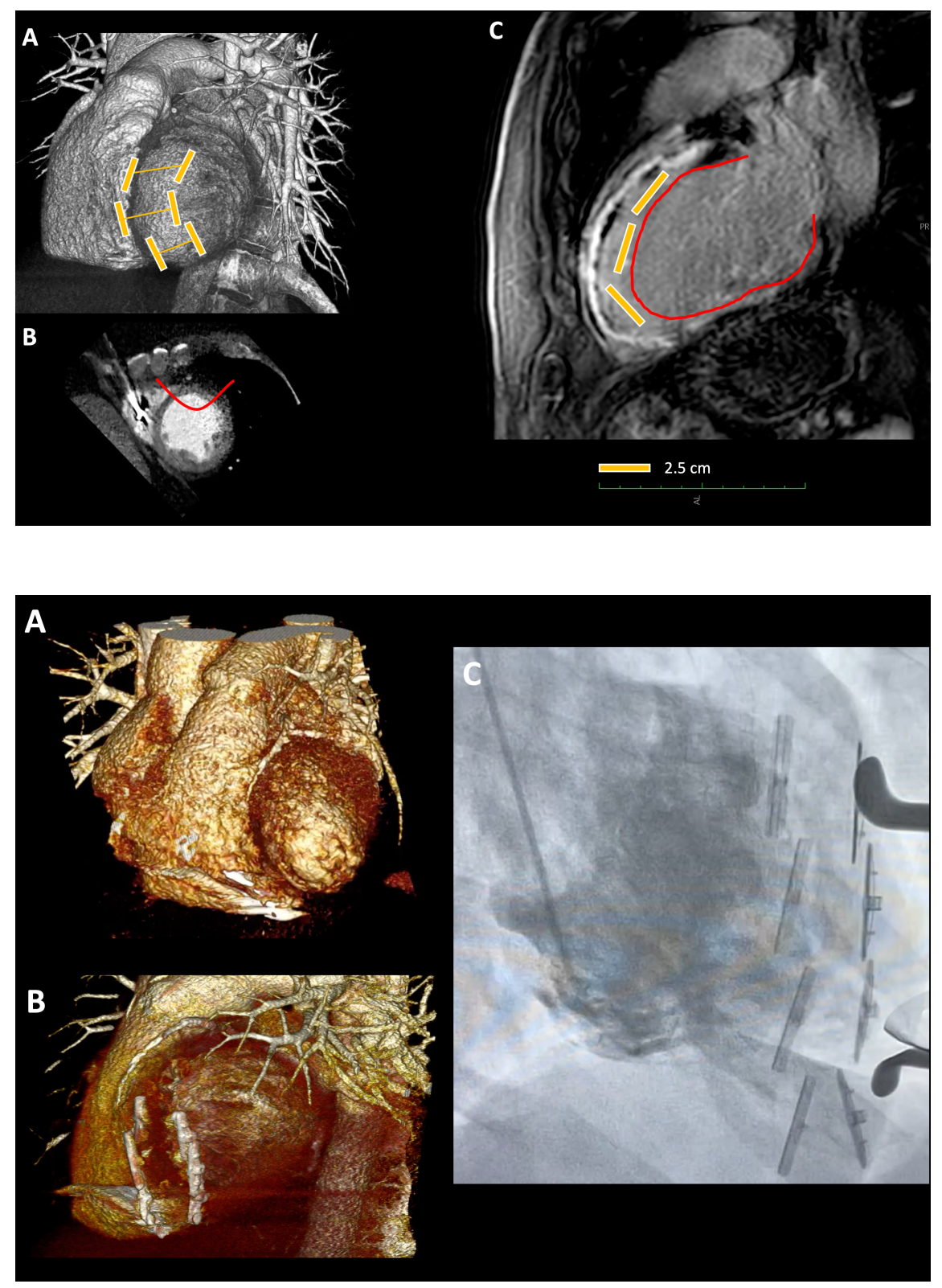